\title{
Brain Connectivity and Network Analysis in Amyotrophic Lateral Sclerosis
}

\author{
Vijay Renga \\ Dartmouth Hitchcock Medical Center, Lebanon, NH 03756, USA \\ Correspondence should be addressed to Vijay Renga; drvijayrenga@gmail.com
}

Received 13 November 2021; Accepted 13 January 2022; Published 7 February 2022

Academic Editor: Mamede de Carvalho

Copyright ( 2022 Vijay Renga. This is an open access article distributed under the Creative Commons Attribution License, which permits unrestricted use, distribution, and reproduction in any medium, provided the original work is properly cited.

\begin{abstract}
Amyotrophic lateral sclerosis (ALS) is a fatal neurodegenerative disease with no effective treatment or cure. ALS is characterized by the death of lower motor neurons (LMNs) in the spinal cord and upper motor neurons (UMNs) in the brain and their networks. Since the lower motor neurons are under the control of UMN and the networks, cortical degeneration may play a vital role in the pathophysiology of ALS. These changes that are not apparent on routine imaging with CT scans or MRI brain can be identified using modalities such as diffusion tensor imaging, functional MRI, arterial spin labelling (ASL), electroencephalogram (EEG), magnetoencephalogram (MEG), functional near-infrared spectroscopy (fNIRS), and positron emission tomography (PET) scan. They can help us generate a representation of brain networks and connectivity that can be visualized and parsed out to characterize and quantify the underlying pathophysiology in ALS. In addition, network analysis using graph measures provides a novel way of understanding the complex network changes occurring in the brain. These have the potential to become biomarker for the diagnosis and treatment of ALS. This article is a systematic review and overview of the various connectivity and networkbased studies in ALS.
\end{abstract}

\section{Introduction}

Amyotrophic lateral sclerosis has an incidence of around 3/ 100,000. No definite etiology has been determined even though various genetic and environmental factors have been attributed [1]. ALS is mostly sporadic, but $5-10 \%$ of cases can be familial [2]. These are associated with SOD1, C9ORF, and other gene mutations that are still being identified. Postmortem studies have shown ALS to be a degenerative disorder of the anterior horn cells in the spinal cord and the cortical neurons, primarily in the motor cortex. The death of the neuronal cell body leads to degeneration of its axons and tracts leading to progressive disability and death [3-5]. In addition to axonal degeneration, there is hyperexcitability of surviving neurons and their networks. Loss of inhibitory neurons, increased glutamatergic activity, and functional reorganization secondary to increased compensation may underlie these changes [5-7]. In addition, interhemispheric inhibition is also impaired in ALS resulting in mirror movements [8].
The pattern of disease onset and rapidity of spread are highly variable. Progression ultimately results in respiratory failure and death, despite artificial means of life support. ALS is part of a spectrum of motor neuron disorders that includes frontotemporal dementia, primary lateral sclerosis, progressive muscular atrophy, and progressive bulbar palsy.

Due to the lack of biomarkers, it is often difficult to diagnose ALS in the early stages of the disease. Misdiagnosis is very common, and patients often go through various expensive and invasive tests before the condition declares itself. Even if diagnosed early, there are too few treatment options if any and that too only in prolonging life for a few months. The lack of biomarkers poses a big hurdle in monitoring response to novel treatments in ALS research.

The role of imaging findings as potential biomarkers gains importance in this scenario. Many structural and functional imaging studies have been performed over the years to assess their utility in ALS. There are no validated measures for clinical use yet. Newer studies using brain connectivity and network analysis look at the brain from a 
broader perspective as a connected network and have the potential to generate biomarkers for ALS. This article highlights the advances in imaging and electrophysiologic techniques to diagnose, assess severity, and predict the progression of ALS based on connectivity parameters and brain network analysis.

\section{Methods}

This article is a literature review of brain connectivity and network analysis studies in ALS (Table 1). Search Terms "ALS" and "Brain Connectivity" yielded 117 articles in Medline. Sixty ALS-specific human studies measuring connectivity and network analysis in ALS were selected for this article (see Figure 1).

The article also provides a primer on routine and specialized imaging in ALS, connectivity analysis, and generation and interpretation of networks using graph measures.

2.1. Clinical Imaging in ALS. Routine imaging studies done as part of clinical workup in ALS include CT scan and MRIs. The utility of the CT scan is limited to identifying focal or global atrophy of the brain and ruling out other pathologies or space-occupying lesions. MRI sequences used for clinical purposes include T1 and T2 weighted sequences, diffusion, and FLAIR images. Rarely, hyperintensities on FLAIR sequences are seen over the motor cortex in ALS patients that at times can be symmetric [9]. While useful as an additional diagnostic finding, these imaging abnormalities are rare and do not have any intrinsic value by themselves in the diagnosis or monitoring of ALS. The high-resolution anatomical MRI images can be further analyzed using voxel-based morphometry (VBM) or surface-based analysis to compute gray matter volumes (GMV). GMV loss is seen in motor and temporal cortices in ALS and frontotemporal dementia (FTD) patients [10]. A compensatory increase in volume in the cerebellar volume has also been reported [11].

2.2. Research Imaging in ALS. There are over 100 billion neurons in the brain that are interconnected through trillions of networks. Such levels of complexity are not measurable by histology or the current level of technology. Instead, imaging techniques such as fMRI, DTI, PET scan, and ANIRS and electrophysiologic techniques such as EEG and MEG can help us generate macroscale representations of these neuronal networks. With advances in imaging techniques, machine learning techniques, and large-scale studies such as the Human Connectome Project, it may become possible for us to define these connections in finer details.

Several brain connectivity studies have been done in ALS with some important and promising findings. However, results have varied and at times contradicted functional connectivity studies. Most studies in ALS have been multimodal with structural studies using high-resolution anatomical MRI and diffusion imaging for white matter tracts and functional connectivity studies using fMRI, EEG, PET, fNIRS, and MEG. The two most commonly used techniques are diffusion imaging and $\mathrm{AMRI}$, which will be described further.

Connectivity studies can be based on structural or functional connectivity. Structural connectivity defines the anatomical connectivity between different regions of the brain. Typically, white matter tracts are defined using diffusion tensor imaging. Anatomical correlates can also be done using anatomical images and surface- or voxel-based techniques. Functional connectivity on the other hand establishes the electrophysiologic or metabolic functional correlates between different regions of the brain. EEG, PET scan, and time series correlates of $\mathrm{MMRI}$ data are used for this purpose.

2.3. Diffusion Imaging. Diffusion imaging captures the spatial diffusion of water molecules along the axonal white matter tracts in different spatial directions assuming a Gaussian distribution. Tensor-based analysis along white matter tracts can provide us fiber direction, axial, radial, and mean diffusivity as well as fractional anisotropy (FA). If the integrity of the myelin sheath is preserved, the fractional anisotropy and axial diffusivity are high, while the radial diffusivity is low. FA is reduced in conditions such as ALS where there is axonal degeneration.

Tractography, by fiber propagation, can generate a visual representation of white matter tracts that can then be further quantified. Deterministic tractography and probabilistic tractography are the two common techniques for generating the tracts. Deterministic tractography uses a set number of seed regions and parameters for propagation in a local fiber direction until a threshold is met for termination. Probabilistic tractography on the other hand takes into consideration propagation in any random direction. Both techniques have pros and cons. Newer techniques such as Q-space imaging may further improve the tracking of fiber bundles bypassing tissue edema and crossing fibers that are limitations of traditional DTI (Figure 2) [12, 13]. Preliminary studies using this technique have shown promising results in ALS that can quantify changes in white matter track volume during interval scans using a track difference paradigm $[14,15]$.

Once tracts are identified, network models can be generated for white matter connectivity. Tract disruptions in ALS are expected in the corticospinal tracts and corpus callosum, but a variety of other non-motor tracts and networks can be affected. Tract-based spatial statistics (TBSS) studies have shown reduced fiber density and reduced mean diffusivity along several white matter tracts in ALS [16-19].

Diffusion tensor imaging studies have consistently shown reduced fractional anisotropy of the corticospinal as well as non-motor tracts for both sporadic and familial ALS [18]. Large studies have also shown a correlation between FA values of corticospinal tracts (CST) with ALS Functional Rating Scale (ALSFRS scores) [20, 21]. Structural connectivity disruptions in the frontal lobe have also shown correlation with cognitive dysfunction in ALS patients [22]. 
TABle 1: Connectivity and network studies done in ALS patients, sorted by year.

2021 network connectivity involving amyotrophic lateral sclerosis and its correlation with disease severity

Functional and structural impairment of transcallosal motor fibers in ALS: a study using transcranial magnetic stimulation, diffusion tensor imaging, and diffusion-weighted spectroscopy Segmental involvement of the corpus callosum in C9orf72associated ALS: a Tract of interestbased DTI study

Ultra-high field (7T) functional magnetic resonance imaging in amyotrophic lateral sclerosis: a Pilot study

Electrical and hemodynamic Neural Functions in People with ALS: an EEG-fNIRS resting-state study

Frontal functional network disruption associated with amyotrophic lateral sclerosis: an fNIRS-based minimum spanning tree analysis

Multimodal longitudinal study of structural brain involvement in amyotrophic lateral sclerosis

Chen et al., 202132 ALS and 45 [59] controls

$\begin{array}{cc}\begin{array}{c}\text { Hübers et al., } \\ 2021\end{array} \text { [8] } & \begin{array}{l}\text { ALS and } 21 \\ \text { controls }\end{array}\end{array}$

Müller et al., $\quad 25$ ALS and 25 2021 [18] controls

Barry et al., $2021 \quad 12$ ALS and 9 [52] controls

Deligani et al., 10 ALS and 9 2020 [64] controls

$\begin{gathered}\text { Borgheal et al., } \\ 2020 \text { [65] }\end{gathered}$
controls

van der Burgh 298 ALS and et al., 2020 [38] 156 controls
sMRI and fMRI:

Independent component analysis and dynamic functional network connectivity dFNC

DTI and TMS: Fractional anisotropy (FA) interhemispheric inhibition (IHI)

DTI: Tractwise fractional anisotropy statistics

sMRI and fMRI:

EEG and fNIRS:

Connectivity study

fNIRS: MST network analysis

sMRI and DTI: cortical thickness, subcortical volumes, and white matter connectivity
ALS patients showed increased dFNC between $\mathrm{DMN}$ and SMN.

ALS patients showed significantly decreased FA in the motor segment of the corpus callosum), and IHI was significantly reduced compared to controls. Regional FA reduction for tracts of the Calloway areas II and III for ALS patients with C9orf72.

Reduced functional connectivity between the superior sensorimotor cortex and bilateral cerebellar lobule

VI in ALS patients. Increased frontoparietal EEG connectivity in the alpha and beta bands and increased interhemispheric and right intrahemispheric fNIRS connectivity in the frontal and prefrontal regions were observed in ALS. Frontal, central, and temporal theta and alpha EEG power decreased in ALS, as did parietal and occipital alpha EEG power, while frontal and parietal hemodynamic spectral power increased in ALS.

Results showed significant between-group differences in several MST topological properties, including leaf fraction, maximum degree, diameter, eccentricity, and degree divergence.

Patients with a C9orf72 mutation showed widespread gray and white matter involvement at baseline and extensive loss of white matter integrity in the connectome over time. In C9orf72negative patients, there was cortical thinning of motor and frontotemporal regions and loss of white matter integrity of connections linked to the motor cortex. 
TABle 1: Continued.

Progression of brain functional connectivity and frontal cognitive dysfunction in ALS
25 ALS

longitudinal
Regional callosal integrity and bilaterality of limb weakness in amyotrophic lateral sclerosis
Tu et al., $2020 \quad 29$ ALS and 25

[33]
Resting-state functional connectivity is decreased globally across the C9orf72 mutation spectrum

Resting-state functional MRI brain signatures of fast disease progression in amyotrophic lateral sclerosis: a retrospective study

Structural and functional brain connectome in motor neuron diseases: a multicenter MRI study

Hippocampal connectivity in amyotrophic lateral sclerosis (ALS): more than Papez circuit impairment

Basia et a. 2020179 ALS and [40] 79 controls
sMRI, FMRI, and DTI: Connectivity and graph analysis

fMRI and DTI: Seed-based connectivity (SBC), voxelbased morphometry (VBM), and tract-based spatial statistics (TBSS)

Involvement of the dentate nucleus in the pathophysiology of amyotrophic lateral sclerosis: a multicenter and multimodal neuroimaging study
Bharti et al., 71 ALS and 56 2020 [85] controls
sMRI, fMRI, and DTI: Seed- and RO-based connectivity and volumetric analysis
FMRI: Resting-state functional connectivity changes
DTI: Probabilistic tractography of the corpus callosum
After six months, ALS patients showed an increased rsFC of the left anterior cingulate, left middle frontal gyrus (MFG), and left superior frontal gyrus within the frontostriatal network, left MFG, left supramarginal gyrus, and right angular gyrus within the left frontoparietal network

In the combined patient group, the most prominent differences in diffusivity metrics were in the rostral body, posterior midbody, and isthmus of the corpus callosum. Loss of corpus callosum integrity was most prominent in the subgroup with unilateral limb weakness at the time of scanning. Both global and connectionspecific decreases in restingstate connectivity were observed, with no substantial reorganization of network hubs.

ALS patients showed reduced functional connectivity in both motor and extramotor networks.

ALS and patients with PLS showed altered structural global network properties, as well as local topologic alterations and decreased structural connectivity in sensorimotor, basal ganglia, frontal, and parietal areas.

Decreased functional connectivity between bilateral hippocampus, bilateral parahippocampal gyri and cerebellum in ALS patients compared with HCs.

DN rsFC was reduced with cerebrum (supplementary motor area, precentral gyrus, frontal, posterior parietal, and temporal), lobule IV, and brain stem, and increased with the parieto-occipital region. 
Abnormal topological organization 2019 of structural covariance networks in amyotrophic lateral sclerosis
Zhang et al., 2019 [86]
60 ALS

patients and

60 controls
Characteristic increases in EEG connectivity correlate with changes of structural MRI in amyotrophic lateral sclerosis
Nasseroleslami 100 ALS and et al., 2019 [87] 34 controls

Patterned functional network disruption in amyotrophic lateral sclerosis
Dukic et al., 201974 ALS and 47 [66] controls
EEG: Functional connectivity using beamformer source analysis
sMRI: Structural covariance networks were studied in ALS patients and control subjects

EEG + sMRi: EEG connectivity
Precentral degeneration and cerebellar compensation in amyotrophic lateral sclerosis: a multimodal MRI analysis
Regional dynamics of the resting brain in amyotrophic lateral sclerosis using fractional amplitude of low-frequency fluctuations and regional homogeneity analyses
Qiu et al., 201960 ALS and 60

[11] controls
sMRI, fMRI, and DTI:

Gray matter volume (GMV), white matter FA, and functional connectivity (FC)
Bueno et al., 20 ALS and 11 2019 [88] controls
fMRI: fractional amplitude of low-frequency fluctuations (fALFF) and regional homogeneity (ReHo)
Structural covariance networks of ALS patients showed an increased path length, clustering coefficient, small world index, and modularity, as well as decreased global efficiency and increased local segregation. Locally, ALS patients showed decreased nodal degree and betweenness in the gyrus rectus and/or Heschl's gyrus and increased betweenness in the supplementary motor area, triangular part of the inferior frontal gyrus, supramarginal gyrus, and posterior cingulate cortex. In addition, in ALS patients, there were more frontal and subcortical hubs than in normal controls. Increased EEG coherence between parietal-frontal scalp regions (in $\gamma$-band) and between bilateral regions over motor areas (in $\theta$-band).

Decreased spectral power in the occipital and temporal $(\delta$ to $\beta$-band), lateral/ orbitofrontal ( $\delta$ - to $\theta$-band) and sensorimotor ( $\beta$-band) regions of the brain in patients with ALS. Furthermore, we show increased comodulation of neural oscillations in the central and posterior $(\delta$-, $\theta$-, and $\gamma \mathrm{l}$-band) and frontal ( $\delta$ and $\gamma$ l-band) regions, as well as decreased synchrony in the temporal and frontal $(\delta$ - to $\beta$-band) and sensorimotor ( $\beta$-band) regions.

Compared with healthy controls, patients with ALS showed decreased GMV in the left precentral gyrus and increased GMV in bilateral cerebellum, decreased FA in the left corticospinal tract and body of corpus callosum, and decreased FC in multiple brain regions, involving bilateral postcentral gyrus, precentral gyrus, and cerebellum anterior lobe, among others.

Decreased fALFF and ReHO in ALS patients compared with HC in widespread cortical regions including motor and sensory regions. 
TABle 1: Continued.

Structural connectivity alterations in amyotrophic lateral sclerosis: a graph theory-based imaging study

Abnormal functional connectivity density in amyotrophic lateral sclerosis

Brain functional networks become more connected as amyotrophic lateral sclerosis progresses: a source level magnetoencephalographic study

Fast progressive lower motor neuron disease is an ALS variant: a two-centre tract of interest-based MRI data analysis

Increased cerebral functional connectivity in ALS: a resting-state magnetoencephalography study

Monitoring value of multimodal magnetic resonance imaging in disease progression of amyotrophic lateral sclerosis: a prospective observational study

Regional thalamic MRI as a marker of widespread cortical pathology and progressive frontotemporal involvement in amyotrophic lateral sclerosis

Fortainer et al., 25 ALS and 26 2019 [77] controls

Li et al., 201838 ALS and 35 [44] controls

Sorrentino et al., 54 ALS and 25 2018 [67] controls

Müller et al., 2018 [35]

65 LMND, 92 controls, and 101 ALS

DTI: Fractional anisotropy maps and voxel-based comparison

24 ALS, 24

Proudfoot et al., 2018 [53]

Shen et al., 2018

controls, 9

PLS, and 15

carriers

MEG: Power fluctuations in neuronal oscillation from distributed cortical parcels

Tu et al., $2018 \quad 20$ ALS and 31
MEG: Betweenness centrality

fMRI: Functional connectivity density ), an method, that quantifies functional connections between a given voxel and all the other voxels in the entire brain

Patients with ALS exhibited global network alterations with decreased global efficiency (Eglob; $p=0.03$ ) and a trend of the reduced whole-brain mean degree $(p=0.05)$ compared to controls.

Patients with ALS were found to have decreased short-range FCD in the primary motor cortex and increased longrange $\mathrm{FCD}$ in the premotor cortex.

The more advanced the disease, the more connected, scale-free, and disassortative the brain networks.

Characteristic alteration patterns along the CST and also in frontal and prefrontal brain areas in LMND patients compared to controls and

ALS. Fast progressing LMND showed substantial involvement, like in ALS, while slow progressors showed less severe alterations. FA of CST is affected in LMND just like ALS.

Increased functional connectivity, particularly from the posterior cingulate cortex, was demonstrated in both patient groups compared to healthy controls. Functional connectivity was increased in the motor areas (fALFF of the right precentral gyri and superior frontal gyri, and ReHo of right precentral gyri) and decreased in the extramotor areas.

Widespread diffusivity alterations in motor and extramotor associated thalamic parcellations. 
The two-year progression of structural and functional cerebral MRI in amyotrophic lateral sclerosis

Unraveling ALS due to SOD1 mutation through the combination of brain and cervical cord MRI
Menke et al., 2018 [90]

16 ALS longitudinal
sMRI, DTI, and FMRI:

$\mathrm{VBM}$ and independent component analysis (ICA)
Agosta et al., 31 ALS and 33 2018 [39]
sMRI, fMRI, DTI: Cortical thickness analysis, diffusion tensor MRI of the corticospinal tracts (CST) and corpus callosum, and resting-state functional connectivity
Aberrant interhemispheric homotopic functional and structural connectivity in amyotrophic lateral sclerosis
Zhang et al., 2017 [46]
Widespread and progressive reductions in gray matter were observed in the precentral gyri and posterior cingulate cortex, as well as progressive local atrophy of the thalamus, caudate, and pallidum bilaterally, and right putamen, hippocampus, and amygdala. The most prominent DTI tract-based changes were in the superior longitudinal fasciculus and corpus callosum. FC decreases were noted between the sensorimotor resting-state network and the frontal pole, between a network comprising both thalami and an area in the visual cortex. Fractional anisotropy showed that sporadic ALS patients had significant CST damage relative to both healthy controls and SOD1-related ALS although the latter showed alterations that were intermediate between controls and sporadic ALS. Functional hyperconnectivity of the motor cortex in the sensorimotor network was observed in patients with sporadic ALS relative to controls.

Extensive reductions of VMHC associated with ALS in brain regions of the precentral and postcentral gyrus, the paracentral lobule, the superior temporal gyrus, the middle cingulate gyrus,
fMRI and DTRI: Voxel mirrored homotopic connectivity (VMHC) and probabilistic fiber tracking
38 ALS and 35 controls. the putamen, and the superior parietal lobules. With DTI, the analysis has also revealed reductions of

interhemispheric structural connectivity through the CC subregions II, III, and V in patients with ALS. Additionally, interhemispheric functional connectivity of the bilateral precentral gyri positively correlated with fractional anisotropy values of the CC subregion III, which structurally connects the bilateral motor cortices. 
TABle 1: Continued.

Brain functional connectome abnormalities in amyotrophic lateral sclerosis are associated with disability and cortical hyperexcitability

Frequency-specific abnormalities of Intrinsic functional connectivity strength among patients with amyotrophic lateral sclerosis: a resting-state fMRI study

Resting-state fMRI correlates of theory of mind impairment in amyotrophic lateral sclerosis

White matter structural network abnormalities underlie executive dysfunction in amyotrophic lateral sclerosis

A large-scale multicenter cerebral diffusion tensor imaging study in amyotrophic lateral sclerosis

Corticoefferent pathways in pure lower motor neuron disease: a diffusion tensor imaging study
Geevasinga et al., 20 ALS and 20 2017 [72] controls
Li et al., 2017
21 ALS and 21
[91]
controls
Trojsi et al., 201721 ALS and 15
[50] controls
fMRI: Resting-state connectivity

fMRI: Functional connectivity strength (FCS)

sMRI, fMRI and TMS

Using threshold tracking transcranial magnetic stimulation (TMS) and functional connectivity

Dimond et al., 18 ALS and 22 2017 [22] controls

Müller et al., 253 ALS and 2016 [92] 189 controls

Rosenbohm 37 LMND and et al., 2016 [31] 53 controls

DTI: FA, AD, RD, MC, diffusion tractography, and whole-brain spatial statistics

Disrupted effective connectivity of the sensorimotor network in amyotrophic lateral sclerosis
Fang et al., 2016

[27]
20 ALS

patients and 21 controls modeling analysis
fMRI: Dynamic causal
Increased functional connectivity in 12 network edges connecting 14 nodes. Connectivity changes in frontal regions are inversely correlated with functional

disability. The mean clustering coefficient was significantly increased in patients with ALS.

ALS patients showed a significantly decreased FCS in the left prefrontal cortex (PFC) and the bilateral superior frontal gyrus. FCS changes in ALS were widespread and frequencydependent.

Decreased connectivity in frontotemporal areas within the main cognitive restingstate networks, including the default mode (DMN), the right and left frontoparietal (R-, L-FPN), and the salience (SLN) networks, in the entire ALS group.

ALS cognitive impaired patients displayed altered

local connectivity and structural integrity in these same frontal regions that correlated with executive dysfunction.

Significant difference in CST, frontal lobe, brainstem, and hippocampus in ALS patients. These changes correlated with the postmortem neuropathologic changes. The analysis demonstrated widespread and characteristic patterns of alterations in patients with LMND, predominantly along the corticospinal tract (CST), with multiple clusters of regional FA reductions.

In ALS patients, significant regional activity alterations in the left primary motor cortex

(M1), the left primary somatosensory cortex (S1) and the right supplementary motor cortex (SMA) are found. Among these regions, spectral DCM revealed a missing closed-loop circuit between the left M1 and the right SMA and lost projection from the right SMA to the left S1 in ALS. 
TABle 1: Continued.

EEG functional network topology is associated with disability in patients with amyotrophic lateral sclerosis

Fraschini et al., 21 ALS and 16 2016 [69] controls

Functional connectivity changes resemble patterns of pTDP-43 pathology in amyotrophic lateral sclerosis

Schulthess et al., 135 ALS and 2016 [55] 56 controls
Increased functional connectivity common to symptomatic amyotrophic lateral sclerosis and those at genetic risk
Menke et al., 12 ALS and 12 2016 [57]
EEG: Functional connectivity using the phase lag index (PLI) and
network topology using the minimum spanning tree (MST)

fMRI and DTI
sMRI, fMRI, and DWI:

Cortical thickness analysis, voxel-based

morphometry, volumetric and shape analyses of subcortical structures,

tract-based spatial statistics of metrics derived from the diffusion tensor, and resting-state functional connectivity

(FC) analyses were performed
Investigating default mode and sensorimotor network connectivity in amyotrophic lateral sclerosis
Chenji et al., 21 ALS and 40 2016 [74]
fMRI: Compared to default mode networks and sensorimotor networks
No significant group differences were observed for the global mean PLI in any frequency band. A significant MST dissimilarity between ALS patients and healthy controls was found in the beta band.

Functional connectivity patterns associated with the motor, brainstem, ventral attention, and default mode/

hippocampal intrinsic connectivity network revealed increased connectivity maps in ALS patients.

Gray matter cortical thickness and shape analysis revealed significant atrophy in patients with ALS (but not psALS) compared with controls in the right primary motor cortex and right caudate.

Comparison of diffusion tensor metrics showed widespread fractional anisotropy and radial diffusivity differences in patients with ALS compared to controls and the psALS group, encompassing parts of the corpus callosum, corticospinal tracts, and superior longitudinal fasciculus. While FC in the resting-state sensorimotor network was similar in psALS and controls, FC between the cerebellum and a network comprising the precuneus, cingulated, and middle frontal lobe was significantly higher in psALS and affected ALS compared to controls.

Significant group differences in resting-state networks

between patients and controls were absent, as was the dependence on the degree of UMN burden. However, DMN connectivity was increased in patients with greater disability and faster progression rate, and SMN connectivity was reduced in those with greater motor impairment. 
TABle 1: Continued.

Occipital cortical gyrification reductions associate with decreased functional connectivity in amyotrophic lateral sclerosis

Widespread temporo-occipital lobe dysfunction in amyotrophic lateral sclerosis

Altered cortical hubs in functional

2015 brain networks in amyotrophic

lateral sclerosis

Cerebro-cerebellar connectivity is increased in primary lateral sclerosis

Functional connectivity changes in resting-state EEG as potential biomarker for amyotrophic lateral sclerosis

Metabolic spatial connectivity in amyotrophic lateral sclerosis as revealed by independent component analysis

Reduced structural connectivity within a prefrontal-motorsubcortical network in amyotrophic lateral sclerosis
Iyer et al., $2015 \quad 18$ ALS and 17

[68] controls

Loawe et al., 64 ALS and 38 2016 [41]

controls

Ma et al., 201520 ALS and 20

[76]

$\mathrm{HC}$

16 PLS

Meoded et al., 2015 [73]

patients and

14 controls
sMRI and fMRI: Surface-

based local gyrification

index (LGI) and seed-

based functional connectivity

FMRI: whole-brain

functional connectivity graph analysis

fMRI: Functional connectivity strength (FCS)

fMRI and DTI: Structural and functional connectivity
EEG: Graph theory measures
Pagani et al., 259 ALS and 2015 [28] 40 controls.
PET: Spatial ICA

Buchanan et al., 30 ALS and 30 sMRI and DTI: Network2015 [32] controls
ALS patients had significantly reduced LGI in the right occipital cortex, and that abnormality in this region was associated with decreased functional connectivity in the bilateral precuneus.

Clusters of reduced functional connectivity were observed in the sensorimotor cortex. High FCS hubs in the prefrontal cortex in ALS versus healthy controls.

PLS patients had 12 regions of increased functional connectivity.

Cross spectral density in the alpha band was higher in patients. In ALS patients, increased degree values of the network nodes were noted in the central and frontal regions in the theta band across seven of the different connectivity maps, among patients, the clustering coefficient in alpha and gamma bands was increased in all regions of the scalp and connectivity were significantly increased $(p=0.02)$. The nodal network showed increased assortativity in the alpha band in the patients group. The clustering coefficient in partial directed connectivity (PDC) showed significantly higher values for patients in alpha, beta, gamma, theta, and delta frequencies.

Eight components were selected as

pathophysiologically meaningful discriminated patients from controls with $99.0 \%$ accuracy.

Impaired motor-frontalsubcortical subnetwork (10 nodes and 12 bidirectional connections), consistent with upper motor neuron pathology. Reduced FA in three of the impaired network connections. 
Additional resources and the 2014 default mode network: evidence of increased connectivity and decreased white matter integrity in amyotrophic lateral sclerosis

Correlation between structural and functional connectivity impairment in amyotrophic lateral sclerosis
Heimrath et al., 2014 [75]

9 ALS patients and 11 controls

fMRI and DTI: Study focused on verbal fluency and attention and the relationship with functional connectivity in the DMN and white matter integrity

Schmidt et al., 64 ALS and 27 2014 [45]
sMRI, DWI, and fMRI

DTI: Whole-brain tractography
Structural brain network imaging shows expanding disconnection of the motor system in amyotrophic lateral sclerosis
Altered motor network functional connectivity in amyotrophic lateral sclerosis: a resting-state functional magnetic resonance imaging study
Divergent brain network connectivity in amyotrophic lateral sclerosis

\section{Agosta et al., 20 ALS and 15} 2013 [42]

\section{ALS}

patients and 12 controls
fMRI: Functional connectivity (FC) of motor cortex
fMRI: Independent component analysis
Patients showed increased functional connectivity in parahippocampal and parietal areas of the non-taskassociated DMN compared to controls. The more pronounced the cognitive deficits, the stronger the increase in functional connectivity in those areas. White matter integrity was reduced in frontal areas in the patients. In conclusion, increased connectivity in the DMN in parahippocampal and parietal areas might represent the recruitment of accessory brain regions to compensate for dysfunctional frontal networks.

(i) The most structurally affected connections considerably overlap with the most functionally impaired connections; (ii) direct connections of the motor cortex are both structurally and functionally more affected than connections at a greater topological distance from the motor cortex; and (iii) there is a strong positive correlation between changes in SC and FC averaged per brain region $(\mathrm{r} 50.44$, $P<0.0001)$.

Demonstrated an expanding subnetwork of affected brain connections over time with a central role for the primary motor regions.

Both decreased and increased within-motor network FC in ALS patients. Increased FC between the bilateral superior parietal lobule and the right anterior inferior cerebellum was found to be correlated with disease severity, with higher FC related to more severe disease.

ALS patients showed a decreased connectivity of the right orbitofrontal cortex and enhanced connectivity of the left precuneus in the default mode network; a decreased connectivity of the left inferior frontal cortex and increased connectivity of the right angular gyrus in the right frontoparietal network; and increased connectivity of the parietal cortex in the left frontoparietal network. 
TABle 1: Continued.

Dysfunctions within limbic-motor networks in amyotrophic lateral sclerosis

Multiple kernel learning captures a systems-level functional connectivity biomarker signature in amyotrophic lateral sclerosis

The utility of independent component analysis and machine learning in the identification of the amyotrophic lateral sclerosis diseased brain

Direct evidence of intra- and interhemispheric corticomotor network degeneration in amyotrophic lateral sclerosis: an automated MRI structural connectivity study
Impaired structural motor

2011 connectome in amyotrophic lateral sclerosis
Feket et al., 201340 ALS and 30

[48] controls $\begin{array}{cc}\text { Passmonti et al., } & 11 \text { ALS and } 12 \\ 2013 \text { [60] } & \text { HC }\end{array}$

Welsh et al., 201332 ALS and 31

[29] controls

Rose et al., 201215 ALS and 15 [20]

$\mathrm{HC}$
fMRI: Intra- and intermotor functional network analysis
fMRI: ICA analysis and support vector machine (SVM) machine learning analysis

sMRI and DWI: Wholebrain diffusion tractography
sMRI and DTI: Fiber Verstraete et al., 35 ALS and 19 2011 [37] controls tracking and network analysis
Sensorimotor functional connectivity changes in amyotrophic lateral sclerosis
Agosta et al., 26 ALS and 15 2011 [58]
fMRI: Resting functional connectivity analysis
Motor network degeneration in amyotrophic lateral sclerosis: a structural and functional connectivity study
Verstaete et al., 12 ALS and 12 2010 [56] controls
Probabilistic diffusion tractography: a potential tool to assess the rate of disease progression in amyotrophic lateral
Ciccarelli et al., 13 ALS and 19 2006 [36] controls
sMRI, DTI, and fMRI:

Whole-brain cortical thickness and diffusion tensor imaging (DTI) of crucial motor tracts and functional connectivity analysis of the motor network based on restingstate fMRI

DTI: Probabilistic tractography, FA analysis of connectivity along CST, and disease progression
ALS patients had greater activation in the PFC areas and altered left amygdala PFC connectivity.

ALS patients had reduced connectivity of both the cortical and subcortical motor areas with non-motor areas, reduced subcortical-cortical motor connectivity, and increased connectivity observed within subcortical motor networks.

Over $71 \%$ accuracy for disease state classification is obtained.

Significant reduction in mean FA within a number of intraand interhemispheric motor pathways in ALS patients is noted.

ALS patient had impaired subnetwork of regions with reduced white matter connectivity around primary motor regions (bilateral precentral gyrus and right paracentral lobule), including secondary motor regions (bilateral caudal middle frontal gyrus and pallidum) as well as high-order hub regions (right posterior cingulate and precuneus). In addition, a significant reduction in overall efficiency and clustering is noted.

ALS patients versus controls showed a significantly increased functional connectivity between the left SMC and the right cingulate cortex, parahippocampal gyrus, and cerebellum-crus II. Functional organization of the motor network was unchanged in patients with ALS compared to healthy controls; however, the level of functional connectedness was significantly correlated with disease progression rate. Patients with increased connectedness had progressive disease courses.

Patients with rapid progression had a significantly lower mean FA. sclerosis rate 


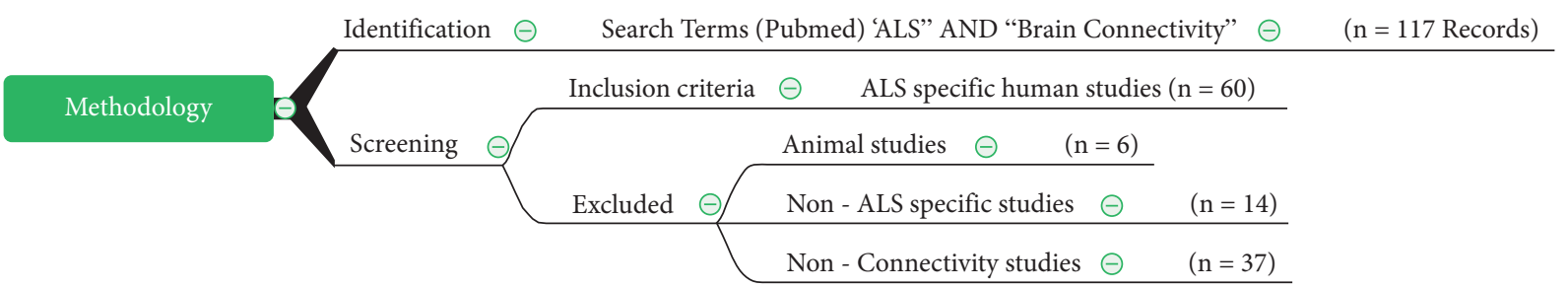

Figure 1: A total of 117 articles were obtained with the search terms. Only ALS-specific human imaging or electrophysiologic studies looking at connectivity were selected for this article. Animal studies, non-ALS studies, and nonconnectivity studies were excluded.

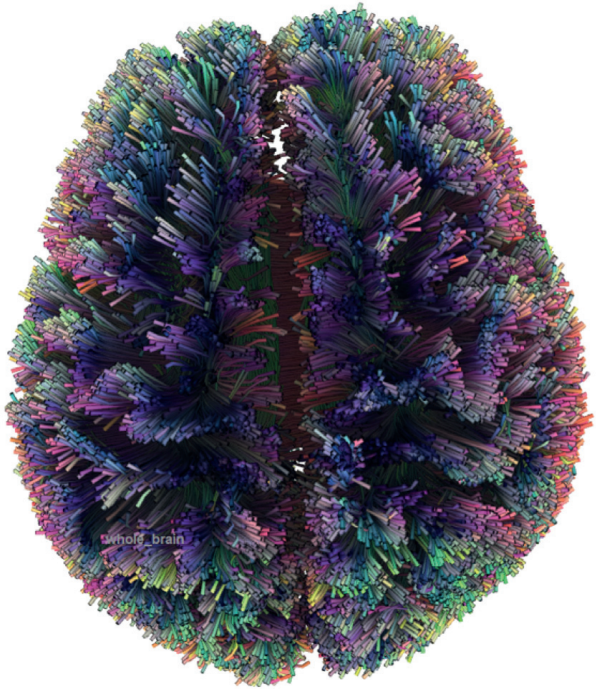

Figure 2: A whole-brain tractography generated based on Q-space diffusion imaging using DSI Studio. Color differences based on fiber direction.

2.4. Functional MRI. Functional MRI (fMRI) measures the changes in regional blood flow during resting or task activation. It uses the blood-oxygen-level-dependent (BOLD) contrast. As regional metabolism causes a shift from oxyhemoglobin to deoxyhemoglobin, there is a change in magnetization properties of blood that can be assessed using MRI. This can be a surrogate for local metabolism and neurovascular coupling [23]. It can also determine regions connected by similar metabolism during rest or activity, helping determine the interconnectedness of various regions using a time series analysis. Connectivity maps and network architecture can be then generated similar to structural connectivity.

\section{Graph Theory and Brain Networks}

Graph theory is a concept that is being increasingly applied to analyze complex brain networks [24]. This concept was first used by Leonard Euler in 1736 to solve the "Konigsberg Bridge" problem. Konigsberg city, which is now Kaliningrad in Russia, is set on both sides of the Pregel River. This created a mainland separated by two islands connected with seven brides. Euler was tasked to conceptualize a walkway throughout the landmasses that interconnected them in such a way that pedestrians needed to cross each bridge only once. Euler made a representation of each landmass as a node and the bridges connecting them as edges. Using early graph theory concepts, he proved that it was not possible to create such a walkway. Various network measures have since been defined to analyze and characterize network architecture. In the modern era, with the developments of transportation networks, the World Wide Web, and social networks, network-based studies became relevant again, and later, its adaptation to neuroscience as the brain is a highly complex network.

Networks consist of nodes and edges. Edges can have directions (directed or undirected) and densities (weighted or unweighted). A simple binary unweighted, undirected graph architecture and various measures are depicted in the graph shown in Figure 2. Graphs can also be depicted using an adjacency matrix that depicts the connections between nodes and edges. It can also be represented in 3D models or 2D connectograms (Figures 3-5).

Brain networks can be created if we parcellate regions of brains into nodes and their connection as edges. Nodes could be anatomical regions, sites of electrode placement, or regions of interest and the "edges" derived from white matter tracts, electrical, hemodynamic, or metabolic connections or time series correlates connecting those nodes. High-resolution structural MRI images are generally used for nodes. Brain regions can be segmented into gray and white matters and then parcelled into distinct regions using anatomical atlases. Various atlases are available that can subdivide the cortical and subcortical regions anywhere from tens to as high as thousands of nodes.

Edges are generated from structural or functional connectivity parameters. Functional connectivity is generally assessed using fMRI time-series data from one region that correlates with other regions in terms of BOLD activity. This could be resting or dynamic. Structural connections are usually derived from diffusion imaging. The diffusion images are corrected for susceptibility and eddy current distortions. White matter edges can be generated using tractography techniques as described earlier.

Once the nodes and edges are defined, networks can be generated and a variety of graph measures are then applied to understand the brain architecture and its disruptions (Figure 4). Network-based statistical analysis can help individual or group of subjects [25]. Large-scale projects such as the Human Connectome Project can provide network databases and templates against which various disease processes could be compared and help understand the network derangement in a variety of diseases [26]. 


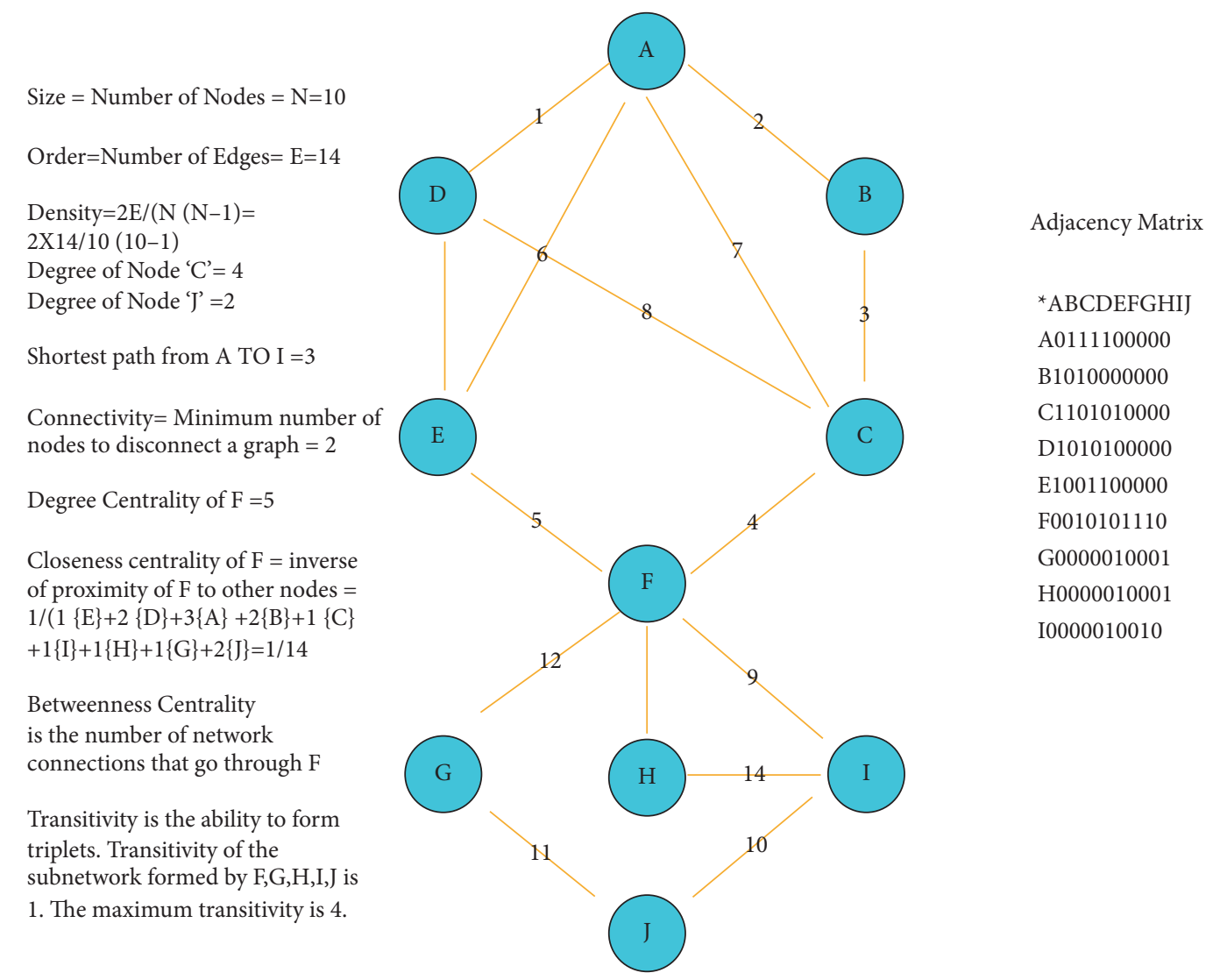

FIGURE 3: A simple unweighted, undirected graph and its adjacency matrix with some commonly used measures.

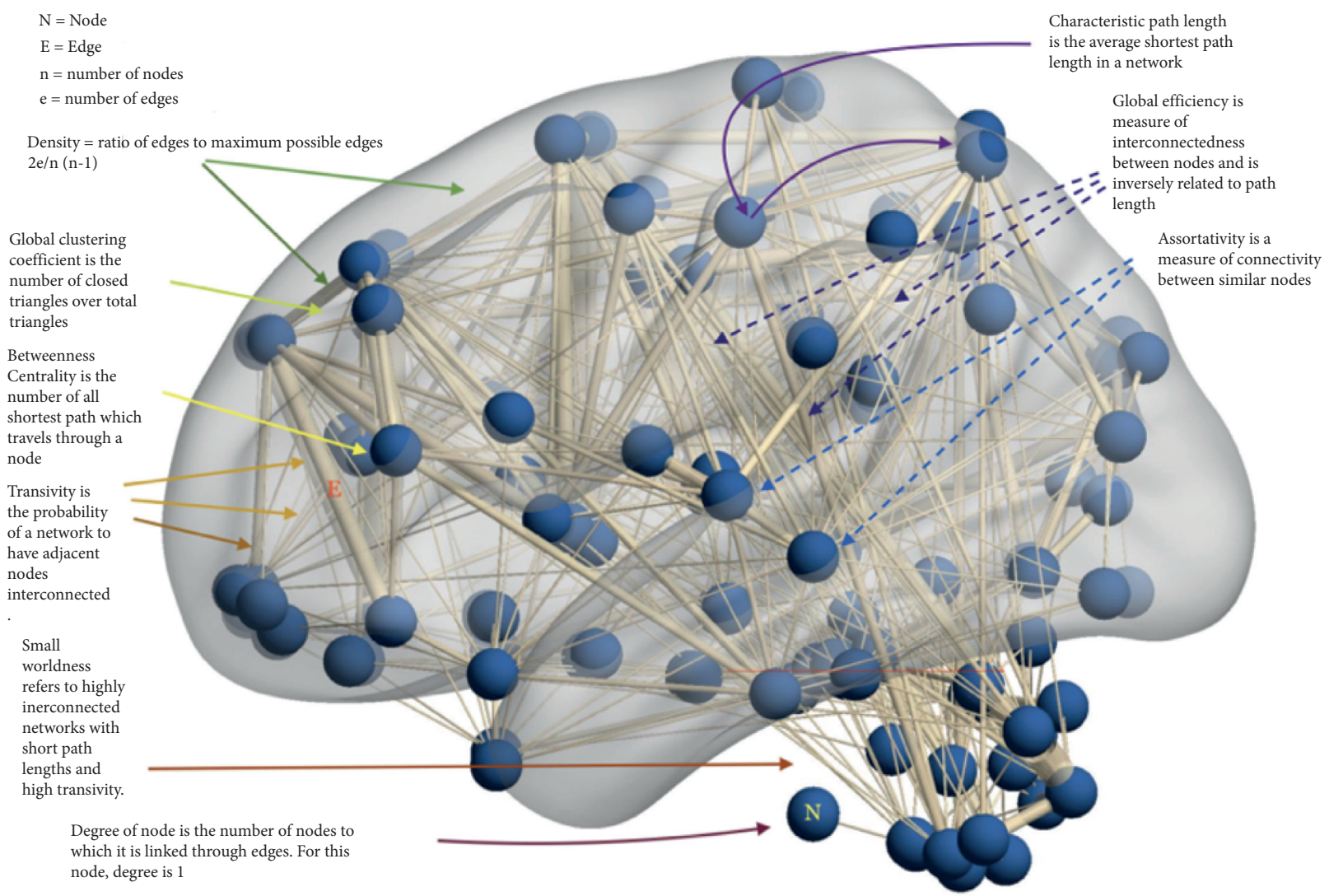

FigURE 4: Various network measures used in graph theory analysis. 

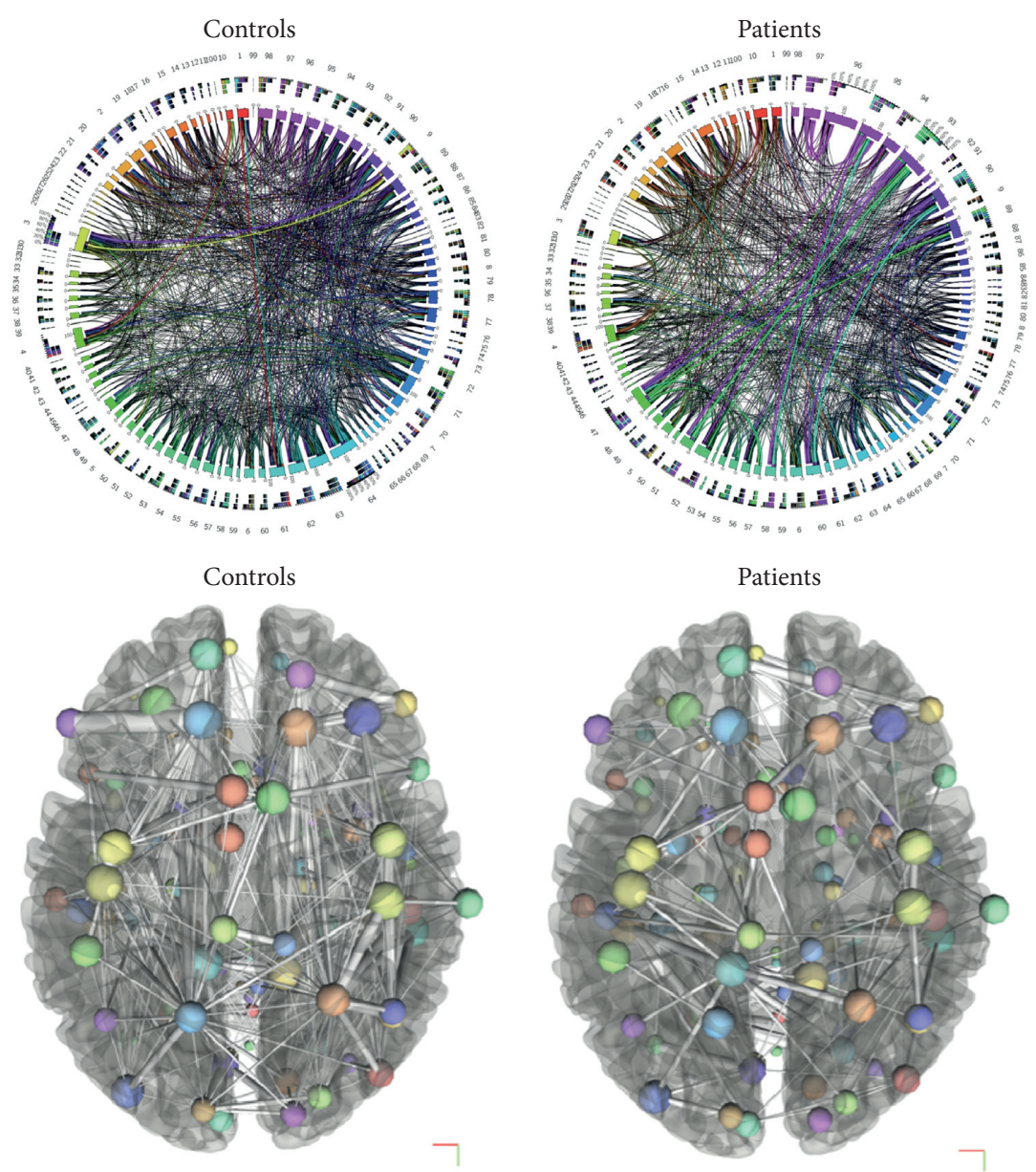

FIGURE 5: Averaged 2D connectograms and 3D structural networks of controls and patients (created with CIRCOS [78] and DSI studio [79])] showing reduced structural network connectivity in ALS. Both $2 \mathrm{D}$ and $3 \mathrm{D}$ models depict a reduced density of white matter connections in ALS patients (R) compared to controls (L). The patterns of connectivity changes can also be visualized.

There are some complex graph measures as shown in (Figure 4). Clustering coefficient is the fraction of triangles around a node and is equivalent to the fraction of the node's neighbors that are neighbors of each other. It is a measure of nodes clustering together. Transitivity measures the probability that the adjacent nodes of a particular node are connected and is closely related to the clustering coefficient. It is calculated as a ratio of the observed number of closed triangles and the maximum possible number of closed triangles in the graph. High transitivity and low path lengths are characteristics of a small-world network. Diameter of a graph refers to the distance towards the maximally eccentric node and radius refers to the minimum eccentricity. The efficiency of a network refers to interconnectedness in a graph network. It can be global or regional. Global efficiency is inversely related to the path length in a network. Assortativity is a measure of similar nodes to be connected. Rich club coefficients refer to well-connected nodes that connect to each other.

\section{Results}

4.1. Connectivity Studies. Brain connectivity studies have utility in analyzing the anatomical and functional derangement in ALS [27]. They have the potential to become biomarkers for ALS $[28,29]$. Longitudinal studies can define the evolution of structural and functional derangements and help us understand the underlying pathophysiology and progression of ALS [30].

Structural connectivity studies have unequivocally shown reduced fractional anisotropy along the motor and non-motor tracts [31-34]. The sites and degree of structural connectivity disruption have correlated with the rate of disease progression $[35,36]$. Intra- and interhemispheric connectivity is also deranged in ALS $[8,20,37]$. This also holds true for genetic ALS with C9ORF mutations $[18,38,39]$. Local connectivity and network parameters vary between different types of motor neuron disease. Primary lateral sclerosis (PLS) and progressive muscular atrophy (PMA) can have differing patterns that can be helpful in identifying the subtype [40].

In contrast to structural connectivity studies, functional connectivity studies have shown differing results with some studies showing lowered and others showing increased functional connectivity in ALS, whether it be sporadic or genetic [41-43]. The pattern of reduced and increased functional connectivity in various regions of the brain also 
differs significantly. Patients with ALS were found to have reduced short-range functional connectivity density in the primary motor cortex and increased long-range connectivity in the premotor cortex [44]. Multimodal studies using anatomical (sMRI), diffusion (DTI), and resting fMRI scans have shown that the more structurally impaired networks overlapped with more functionally impaired connections [45]. Voxel mirrored homotopic interhemispheric connectivity of structural and functional networks involving the corpus callosum has shown reduced functional connectivity [46]. Decreased functional connectivity has been reported in the premotor cortex, corpus callosum, hippocampus, and cerebellar regions $[11,47-52]$. In contrast to these findings, more studies have shown increased functional connectivity in ALS patients, even in regions with reduced structural connectivity [40, 53-58]. Dynamic connectivities of default motor networks and sensorimotor networks have increased connectivity in ALS [59]. Functional MRI (fMRI) studies using motor task activation have shown increased activation clusters in ALS patients compared to controls for the same task. Higher activation was seen in the prefrontal cortex in ALS patients compared to controls [60-63]. Such hyperconnectivity has been the predominant finding in studies using EEG, fNIRS, and MEG, even though some differences were noted here as well $[53,64-69]$. Such differences in results can be explained by a heterogeneous progression of functional connectivity changes in ALS occurring at different stages of disease evolution when the patients were studied [44]. Functional hyperconnectivity or hyperexcitability may be the result of an intrinsic pathophysiologic process or a compensatory response to weakened musculature. Progressive increase in functional connectivity in frontoparietal and frontostriatal networks has been shown in longitudinal studies [70]. This increase in functional connectivity has also been shown to correlate with the severity of the disease process $[67,71-73]$. Studies interrogating specific resting-state networks of the brain have identified increased connectivity in the default mode network and reduced connectivity in sensorimotor network at the same time and both these correlated to the severity of the disease process $[74,75]$. While there are regions of increased and decreased regional functional connectivity in ALS, the global pattern across various studies leans towards increased functional connectivity, suggestive of the hyperexcitable cortex [59].

Despite several different studies, the functional connectivity changes in ALS remain complex and poorly defined. More longitudinal studies are needed to understand the evolution of functional connectivity disruption in ALS.

4.2. Network-Based Studies in ALS. Few studies have looked at network disruption in ALS using graph theory. These were mostly done using structural MRI (sMRI), fMRI, and DTI. Few EEG, MEG, and fNIRS studies have also been conducted.

Analysis of graph metrics using global measures in C9ORF mutation carriers showed lower global network density than healthy controls [43]. The mean clustering coefficient has been shown to be significantly higher in ALS patients compared to controls [68]. Resting-state functional networks in ALS versus healthy controls using a voxel-level approach showed significant differences in degree centrality in some regions in ALS [71]. The nodal degree in the left superior frontal region has also correlated with the ALSFRS-r scores.

Larger studies using high-resolution T1 weighted anatomical images and structural covariance networks showed significant increases in path length, clustering coefficient, small-world index, and local efficiency in ALS patients. At the same time, there was a significant decrease in global efficiency at several network densities in ALS patients compared to controls. Modularity was higher in ALS patients for several network densities. The modularity increase indicates fragmentation of brain architecture into more tightly clustered modules with poor intermodal communication in ALS patients. Changes were also noted in regional networks and network hubs. Higher functional connectivity strength hubs were seen in ALS patients compared to healthy controls [76]. Nodal betweenness was also increased in several regions in ALS suggesting an increase in information transfer across the available nodes suggestive of an increased "traffic."

Significantly different networks connecting various regions of the brain have been found in ALS patients. Such findings are important to identify heterogeneously progressing vulnerable networks in ALS. Structural network analysis of these deranged networks showed reduced local and global efficiency in ALS patients compared to controls $[40,77]$. Functional network studies in ALS have shown differing results like the functional connectivity studies. Verstraete et al. studied diffusion MRI-derived white matter structural connectome in patients with ALS. Subnetworks of significance were identified using network-based statistics $[45,56]$. They detected impaired subnetworks within both motor cortex and distant regions, most of which were involved in motor control. In the impaired networks, they detected low network efficiency and density. A connectivity study done by the same group in ALS showed increased functional connectedness in ALS-affected structural networks [56]. A similar finding of impaired reduced structural connectivity within the prefrontal-motor-subcortical white matter network has been demonstrated using network-based statistics.

Electrophysiologic studies have also been useful in identifying the functional connectivity and network parameters in ALS patients. EEG data in ALS patients showed an increased spectral density of alpha bands. Clustering coefficients in alpha and gamma bands were increased in all regions of the scalp. Overall connectivity was increased in ALS patients with increased assortativity in the alpha band [68]. Resting-state MEG study in ALS also showed increased functional connectivity in the posterior cingulate cortex in ALS patients [53]. Functional network reorganization is perturbed in ALS, which has been shown to correlate with the disability $[65,69]$.

We studied differences in global structural and functional connectivity patterns between eight ALS patients and eight age-matched healthy control data from online sources in a pilot connectivity in ALS (CoALS) study [54]. Global structural measures computed were density, clustering 

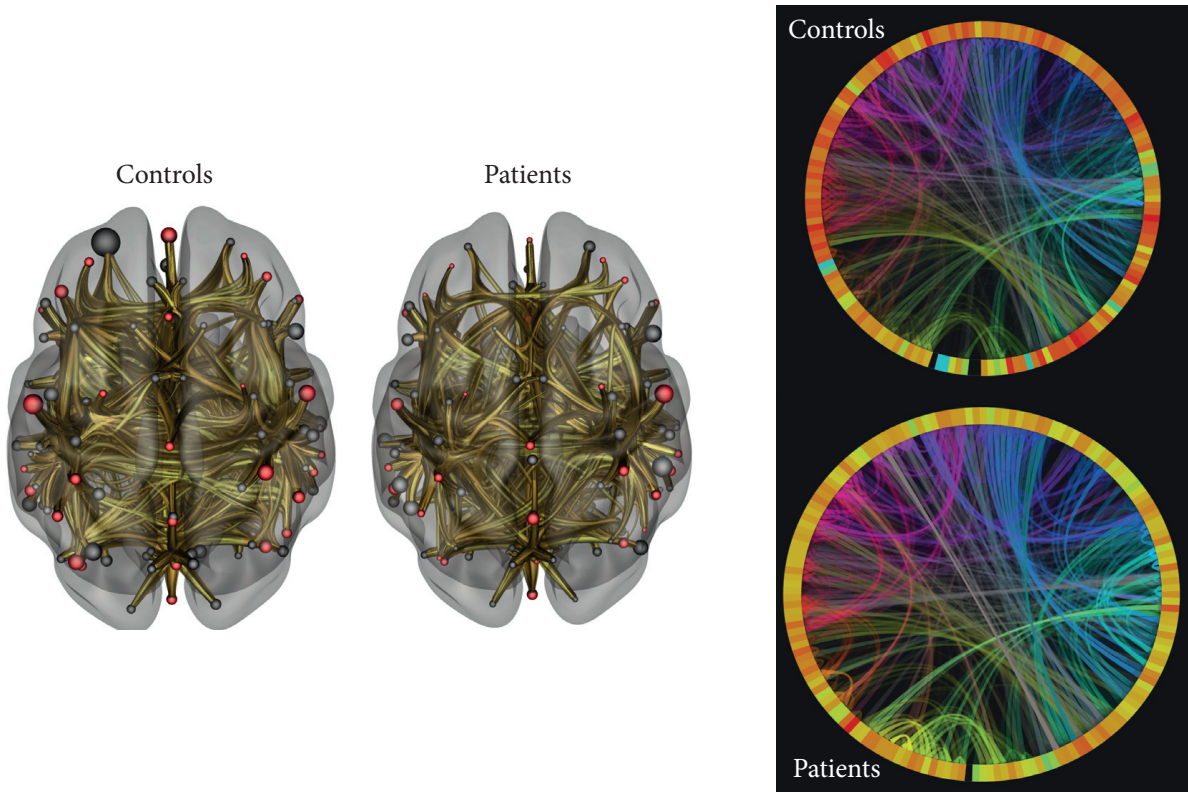

FIGURE 6: Averaged functional 2D connectogram and 3D networks of controls and patients (created using CONN Toolbox [80]). While 3D functional connection density appears similar, the pattern difference can be seen on the 2D connectograms. Analysis revealed increased interconnectedness (global efficiency) in ALS patients (not visualized in this picture).

coefficient, transitivity, characteristic path length, small worldness, global efficiency, diameter, radius, assortativity, and rich club coefficients. For functional MRI global efficiency, local efficiency, betweenness centrality, average path length, clustering coefficient, and degree were compared between the groups. Structural network density was found to be significantly lower in ALS patients compared to control subjects (Figure 5). At the same time, the global functional efficiency was found to be significantly increased in ALS patients compared to control subjects (Figure 6). Other measures did not differ significantly. This finding is in alignment with our understanding of network architecture breakdown and hyperconnectivity in ALS. Bigger studies are underway to validate these findings and explore the network architecture further.

\section{Conclusion}

Brain connectivity and network analysis provide us with a novel and non-invasive approach to trace the progression of highly complex structural and functional derangements in ALS. Connectivity and network measures have shown correlation with disease progression and severity. While structural studies have unequivocally shown degrading connectivity and network architecture, functional studies have differed. These differences could be related to evolutionary changes occurring in a nonuniform pattern similar to the clinical presentation of ALS. More longitudinal studies are necessary to clarify this process.

The studies done so far have indicated a "traffic-jam" type pattern in the brain of ALS patients, where structural "road" networks are disrupted with an increase in functional connectedness or "traffic." This functional interconnectedness and hyperexcitability seem to increase with the disease progression [67]. Whether this hyperexcitability is a response to structural disruption, or an intrinsic pathophysiologic process remains unclear $[5,81]$. It can be hypothesized that a potential treatment of ALS would be the one that would either stop the structural network degradation or reduce the functional hyperconnectivity or both.

As we become more proficient in characterizing the underlying network changes with more standardized methodologies and automated analysis, we may be able to further characterize the intricate changes occurring in various stages of ALS [29, 82]. Vulnerable subnetworks, which are pathways of ALS progression, need to be isolated since those could become potential targets for treatment. Changing excitability of brain tissue using techniques like brain stimulation may be helpful in ALS [83].

Multimodal brain MRI findings using DTI and fMRI have already been proposed to have biomarker value for diagnosis and stratification in ALS [84]. Adding network analysis to these techniques may provide us with additional information to clarify and quantify responses to new and emerging treatments. With further refinements in connectivity analysis and a deeper understanding of the network topology, we may finally be able to define a network fingerprint for ALS that will have great implications in the diagnosis and treatment of ALS.

\section{Conflicts of Interest}

The author declares that there are no conflicts of interest.

\section{References}

[1] A. S. Andrew, W. G. Bradley, D. Peipert et al., "Risk factors for amyotrophic lateral sclerosis: a regional United States casecontrol study," Muscle \& Nerve, vol. 63, no. 1, pp. 52-59, 2021. 
[2] A. Chiò, A. Calvo, C. Moglia, L. Mazzini, G. Mora, and PARALS study group, "Phenotypic heterogeneity of amyotrophic lateral sclerosis: a population based study," Journal of Neurology Neurosurgery and Psychiatry, vol. 82, pp. 740-746, 2011.

[3] J. A. Kiernan and A. J. Hudson, "Changes in sizes of cortical and lower motor neurons in amyotrophic lateral sclerosis," Brain, vol. 114, no. 2, pp. 843-853, 1991.

[4] K. Nihei, A. C. McKee, and N. W. Kowall, "Patterns of neuronal degeneration in the motor cortex of amyotrophic lateral sclerosis patients," Acta Neuropathologica, vol. 86, no. 1, pp. 55-64, 1993.

[5] N. Kohara, "Abnormal hyperexcitability in ALS," Rinsho Shinkeigaku, vol. 39, pp. 61-64, 1999.

[6] P. Menon, M. C. Kiernan, and S. Vucic, "Cortical hyperexcitability precedes lower motor neuron dysfunction in ALS," Clinical Neurophysiology, vol. 126, no. 4, pp. 803-809, 2015.

[7] M. A. J. Van den Bos, M. Higashihara, N. Geevasinga, P. Menon, M. C. Kiernan, and S. Vucic, "Imbalance of cortical facilitatory and inhibitory circuits underlies hyperexcitability in ALS," Neurology, vol. 91, no. 18, pp. e1669-e1676, 2018.

[8] A. Hübers, B. Böckler, A. Abaei et al., "Functional and structural impairment of transcallosal motor fibres in ALS: a study using transcranial magnetic stimulation, diffusion tensor imaging, and diffusion weighted spectroscopy," Brain Imaging and Behavior, vol. 15, pp. 748-757, 2021.

[9] L. Zhang, A. M. Ulu, R. D. Zimmerman, M. T. Lin, M. Rubin, and M. F. Beal, "The diagnostic utility of FLAIR imaging in clinically verified amyotrophic lateral sclerosis," Journal of Magnetic Resonance Imaging, vol. 17, no. 5, pp. 521-527, 2003.

[10] P. Bede, T. Omer, E. Finegan et al., "Connectivity-based characterisation of subcortical grey matter pathology in frontotemporal dementia and ALS: a multimodal neuroimaging study," Brain Imaging and Behavior, vol. 12, no. 6, pp. 1696-1707, 2018.

[11] T. Qiu, Y. Zhang, X. Tang et al., "Precentral degeneration and cerebellar compensation in amyotrophic lateral sclerosis: a multimodal MRI analysis," Human Brain Mapping, vol. 40, pp. 3464-3474, 2019.

[12] F.-C. Yeh, V. J. Wedeen, and W.-Y. I. Tseng, "Generalized $\$\{$ q\}\$-sampling imaging," IEEE Transactions on Medical Imaging, vol. 29, no. 9, pp. 1626-1635, 2010.

[13] F.-C. Yeh, T. D. Verstynen, Y. Wang, J. C. Fernández-Miranda, and W.-Y. I. Tseng, "Deterministic diffusion fiber tracking improved by quantitative anisotropy," PLoS One, vol. 8, no. 11, Article ID e80713, 2013.

[14] K. Abhinav, F. C. Yeh, A. El-Dokla et al., "Use of diffusion spectrum imaging in preliminary longitudinal evaluation of amyotrophic lateral sclerosis: development of an imaging biomarker," Frontiers in Human Neuroscience, vol. 8, p. 270, 2014.

[15] F.-C. Yeh, I. M. Zaydan, V. R. Suski et al., "Differential tractography as a track-based biomarker for neuronal injury," NeuroImage, vol. 202, Article ID 116131, 2019.

[16] S. M. Smith, M. Jenkinson, H. Johansen-Berg et al., "Tractbased spatial statistics: voxelwise analysis of multi-subject diffusion data," NeuroImage, vol. 31, no. 4, pp. 1487-1505, 2006.

[17] A. R. Alruwaili, K. Pannek, A. Coulthard, R. Henderson, N. D. Kurniawan, and P. McCombe, "A combined tract-based spatial statistics and voxel-based morphometry study of the first MRI scan after diagnosis of amyotrophic lateral sclerosis with subgroup analysis," Journal of Neuroradiology, vol. 45, no. 1, pp. 41-48, 2018.
[18] H.-P. Müller, D. Lulé, F. Roselli, A. Behler, A. C. Ludolph, and J. Kassubek, "Segmental involvement of the corpus callosum in C9orf72-associated ALS: a tract of interest-based DTI study," Therapeutic Advances in Chronic Disease, vol. 12, Article ID 20406223211002970, 2021.

[19] O. Ciccarelli, T. E. Behrens, H. Johansen-Berg et al., "Investigation of white matter pathology in ALS and PLS using tract-based spatial statistics," Human Brain Mapping, vol. 30, no. 2, pp. 615-624, 2009.

[20] S. Rose, K. Pannek, C. Bell et al., "Direct evidence of intra- and interhemispheric corticomotor network degeneration in amyotrophic lateral sclerosis: an automated MRI structural connectivity study," NeuroImage, vol. 59, no. 3, pp. 2661-2669, 2012.

[21] E. Verstraete, J. H. Veldink, L. H. van den Berg, and M. P. van den Heuvel, "Structural brain network imaging shows expanding disconnection of the motor system in amyotrophic lateral sclerosis," Human Brain Mapping, vol. 35, no. 4, pp. 1351-1361, 2014.

[22] D. Dimond, A. Ishaque, S. Chenji et al., "White matter structural network abnormalities underlie executive dysfunction in amyotrophic lateral sclerosis," Human Brain Mapping, vol. 38, no. 3, pp. 1249-1268, 2017.

[23] D. G. Nair, "About being BOLD," Brain Research Reviews, vol. 50, no. 2, pp. 229-243, 2005.

[24] M. Rubinov and O. Sporns, "Complex network measures of brain connectivity: uses and interpretations," NeuroImage, vol. 52, no. 3, pp. 1059-1069, 2010.

[25] A. Zalesky, A. Fornito, and E. T. Bullmore, "Network-based statistic: identifying differences in brain networks," NeuroImage, vol. 53, no. 4, pp. 1197-1207, 2010.

[26] D. C. Van Essen, S. M. Smith, D. M. Barch, T. E. J. Behrens, E. Yacoub, and K. Ugurbil, "The Wu-Minn human connectome project: an overview," NeuroImage, vol. 80, pp. 62-79, 2013.

[27] X. Fang, Y. Zhang, Y. Wang et al., "Disrupted effective connectivity of the sensorimotor network in amyotrophic lateral sclerosis," Journal of Neurology, vol. 263, no. 3, pp. 508-516, 2016.

[28] M. Pagani, J. Öberg, F. De Carli et al., "Metabolic spatial connectivity in amyotrophic lateral sclerosis as revealed by independent component analysis," Human Brain Mapping, vol. 37, no. 3, pp. 942-953, 2016.

[29] R. C. Welsh, L. M. Jelsone-Swain, and B. R. Foerster, "The utility of independent component analysis and machine learning in the identification of the amyotrophic lateral sclerosis diseased brain," Frontiers in Human Neuroscience, vol. 7, 2013.

[30] R. A. L. Menke, M. Proudfoot, K. Talbot, and M. R. Turner, "The two-year progression of structural and functional cerebral MRI in amyotrophic lateral sclerosis," NeuroImage: Clinical, vol. 17, pp. 953-961, 2018.

[31] A. Rosenbohm, H.-P. Müller, A. Hübers, A. C. Ludolph, and J. Kassubek, "Corticoefferent pathways in pure lower motor neuron disease: a diffusion tensor imaging study," Journal of Neurology, vol. 263, no. 12, pp. 2430-2437, 2016.

[32] C. R. Buchanan, L. D. Pettit, A. J. Storkey, S. Abrahams, and M. E. Bastin, "Reduced structural connectivity within a prefrontal-motor-subcortical network in amyotrophic lateral sclerosis," Journal of Magnetic Resonance Imaging, vol. 41, no. 5, pp. 1342-1352, 2015.

[33] S. Tu, C. Wang, R. A. L. Menke et al., "Regional callosal integrity and bilaterality of limb weakness in amyotrophic lateral sclerosis," Amyotrophic Lateral Sclerosis and Frontotemporal Degeneration, vol. 21, no. 5-6, pp. 396-402, 2020. 
[34] S. Tu, R. A. L. Menke, K. Talbot, M. C. Kiernan, and M. R. Turner, "Regional thalamic MRI as a marker of widespread cortical pathology and progressive frontotemporal involvement in amyotrophic lateral sclerosis," Journal of Neurology, Neurosurgery \& Psychiatry, vol. 89, no. 12, pp. 1250-1258, 2018.

[35] H. P. Müller, F. Agosta, N. Riva et al., "Fast progressive lower motor neuron disease is an ALS variant: a two-centre tract of interest-based MRI data analysis," NeuroImage. Clinical, vol. 17, pp. 145-152, 2018.

[36] O. Ciccarelli, T. E. Behrens, and D. R. Altmann, "Probabilistic diffusion tractography: a potential tool to assess the rate of disease progression in amyotrophic lateral sclerosis," Brain, vol. 129, no. 7, pp. 1859-1871, 2006.

[37] E. Verstraete, J. H. Veldink, R. C. W. Mandl, L. H. van den Berg, and M. P. van den Heuvel, "Impaired structural motor connectome in amyotrophic lateral sclerosis," PLoS One, vol. 6, no. 9, Article ID e24239, 2011.

[38] H. K. van der Burgh, H.-J. Westeneng, R. Walhout et al., "Multimodal longitudinal study of structural brain involvement in amyotrophic lateral sclerosis," Neurology, vol. 94, no. 24, pp. e2592-e2604, 2020.

[39] F. Agosta, E. G. Spinelli, I. V. Marjanovic et al., "Unraveling ALS due to SOD1 mutation through the combination of brain and cervical cord MRI," Neurology, vol. 90, no. 8, pp. e707-e716, 2018.

[40] S. Basaia, F. Agosta, C. Cividini et al., "Structural and functional brain connectome in motor neuron diseases," Neurology, vol. 95, no. 18, pp. e2552-e2564, 2020.

[41] K. Loewe, J. Machts, J. Kaufmann et al., "Widespread temporo-occipital lobe dysfunction in amyotrophic lateral sclerosis," Scientific Reports, vol. 7, no. 1, p. 40252, 2017.

[42] F. Agosta, E. Canu, P. Valsasina et al., "Divergent brain network connectivity in amyotrophic lateral sclerosis," Neurobiology of Aging, vol. 34, no. 2, pp. 419-427, 2013.

[43] R. F. Smallwood Shoukry, M. G. Clark, and M. K. Floeter, "Resting state functional connectivity is decreased globally across the C9orf72 mutation spectrum," Frontiers in Neurology, vol. 11, Article ID 598474, 2020.

[44] W. Li, J. Zhang, C. Zhou et al., "Abnormal functional connectivity density in amyotrophic lateral sclerosis," Frontiers in Aging Neuroscience, vol. 10, p. 215, 2018.

[45] R. Schmidt, E. Verstraete, M. A. Reus, J. H. Veldink, L. H. den Berg, and M. P. den Heuvel, "Correlation between structural and functional connectivity impairment in amyotrophic lateral sclerosis," Human Brain Mapping, vol. 35, no. 9, pp. 4386-4395, 2014.

[46] J. Zhang, B. Ji, J. Hu et al., “Aberrant interhemispheric homotopic functional and structural connectivity in amyotrophic lateral sclerosis," Journal of Neurology Neurosurgery and Psychiatry, vol. 88, pp. 369-371, 2017.

[47] F. Trojsi, F. Di Nardo, G. Caiazzo et al., "Hippocampal connectivity in amyotrophic lateral sclerosis (ALS): more than papez circuit impairment," Brain Imaging and Behavior, vol. 15, no. 4, pp. 2126-2138, 2021.

[48] T. Fekete, N. Zach, L. R. Mujica-Parodi, and M. R. Turner, "Multiple Kernel learning captures a systems-level functional connectivity biomarker signature in amyotrophic lateral sclerosis," PLoS One, vol. 8, pp. 1-8, 2013.

[49] Y. Zhang, T. Fang, Y. Wang et al., "Occipital cortical gyrification reductions associate with decreased functional connectivity in amyotrophic lateral sclerosis," Brain Imaging and Behavior, vol. 11, no. 1, pp. 1-7, 2017.

[50] F. Trojsi, F. Di Nardo, G. Santangelo et al., "Resting state fMRI correlates of theory of mind impairment in amyotrophic lateral sclerosis," Cortex, vol. 97, pp. 1-16, 2017.
[51] F. Trojsi, F. Di Nardo, M. Siciliano et al., "Resting state functional MRI brain signatures of fast disease progression in amyotrophic lateral sclerosis: a retrospective study," Amyotrophic Lateral Sclerosis and Frontotemporal Degeneration, vol. 22, no. 1-2, pp. 117-126, 2021.

[52] R. L. Barry, S. Babu, S. A. Anteraper et al., "Ultra-high field (7T) functional magnetic resonance imaging in amyotrophic lateral sclerosis: a pilot study," NeuroImage: Clinical, vol. 30, Article ID 102648, 2021.

[53] M. Proudfoot, G. L. Colclough, A. Quinn et al., "Increased cerebral functional connectivity in ALS," Neurology, vol. 90, no. 16, pp. e1418-e1424, 2018.

[54] V. Renga, "Connectivity in ALS (CoALS) - a pilot study (359)," Neurology. Wolters Kluwer Health, Inc. on behalf of the American Academy of Neurology, vol. 94, 2020.

[55] I. Schulthess, M. Gorges, H.-P. Müller et al., "Functional connectivity changes resemble patterns of pTDP-43 pathology in amyotrophic lateral sclerosis," Scientific Reports, vol. 6, no. 1, Article ID 38391, 2016.

[56] E. Verstraete, M. P. van den Heuvel, J. H. Veldink et al., "Motor network degeneration in amyotrophic lateral sclerosis: a structural and functional connectivity study," PLoS One, vol. 5, no. 10, Article ID e13664, 2010.

[57] R. A. L. Menke, M. Proudfoot, J. Wuu et al., "Increased functional connectivity common to symptomatic amyotrophic lateral sclerosis and those at genetic risk," Journal of Neurology, Neurosurgery \& Psychiatry, vol. 87, no. 6, pp. 580-588, 2016.

[58] F. Agosta, P. Valsasina, M. Absinta et al., "Sensorimotor functional connectivity changes in amyotrophic lateral sclerosis," Cerebral Cortex, vol. 21, no. 10, pp. 2291-2298, 2011.

[59] H.-J. Chen, Z.-Y. Zou, X.-H. Zhang, J.-Y. Shi, N.-X. Huang, and Y.-J. Lin, "Dynamic changes in functional network connectivity involving amyotrophic lateral sclerosis and its correlation with disease severity," Journal of Magnetic Resonance Imaging, vol. 54, no. 1, pp. 239-248, 2021.

[60] L. Passamonti, F. Fera, A. Tessitore et al., "Dysfunctions within limbic-motor networks in amyotrophic lateral sclerosis," Neurobiology of Aging, vol. 34, no. 11, pp. 2499-2509, 2013.

[61] K. Kollewe, T. F. Münte, A. Samii, R. Dengler, S. Petri, and B. Mohammadi, "Patterns of cortical activity differ in ALS patients with limb and/or bulbar involvement depending on motor tasks," Journal of Neurology, vol. 258, no. 5, pp. 804-810, 2011.

[62] B. Mohammadi, K. Kollewe, A. Samii, K. Krampfl, R. Dengler, and T. F. Münte, "Decreased brain activation to tongue movements in amyotrophic lateral sclerosis with bulbar involvement but not Kennedy syndrome," Journal of Neurology, vol. 256, no. 8, pp. 1263-1269, 2009.

[63] B. Mohammadi, K. Kollewe, A. Samii, R. Dengler, and T. F. Münte, "Functional neuroimaging at different disease stages reveals distinct phases of neuroplastic changes in amyotrophic lateral sclerosis," Human Brain Mapping, vol. 32, no. 5, pp. 750-758, 2011.

[64] R. J. Deligani, S. I. Hosni, S. B. Borgheai et al., "Electrical and hemodynamic neural Functions in people with ALS: an EEGfNIRS resting-state study," IEEE Transactions on Neural Systems and Rehabilitation Engineering, vol. 28, no. 12, pp. 3129-3139, 2020.

[65] S. B. Borgheai, J. McLinden, K. Mankodiya, and Y. Shahriari, "Frontal functional network disruption associated with amyotrophic lateral sclerosis: an fNIRS-based minimum spanning tree analysis," Frontiers in Neuroscience, vol. 14, Article ID 613990, 2020. 
[66] S. Dukic, R. McMackin, T. Buxo et al., "Patterned functional network disruption in amyotrophic lateral sclerosis," Human Brain Mapping, vol. 40, no. 16, pp. 4827-4842, 2019.

[67] P. Sorrentino, R. Rucco, F. Jacini et al., "Brain functional networks become more connected as amyotrophic lateral sclerosis progresses: a source level magnetoencephalographic study," NeuroImage: Clinical, vol. 20, pp. 564-571, 2018.

[68] P. M. Iyer, C. Egan, M. Pinto-Grau et al., "Functional connectivity changes in resting-state EEG as potential biomarker for amyotrophic lateral sclerosis," PLoS One, vol. 10, no. 6, Article ID e0128682, 2015.

[69] M. Fraschini, M. Demuru, A. Hillebrand et al., "EEG functional network topology is associated with disability in patients with amyotrophic lateral sclerosis," Scientific Reports, vol. 6, no. 1, p. 38653, 2016.

[70] V. Castelnovo, E. Canu, D. Calderaro et al., "Progression of brain functional connectivity and frontal cognitive dysfunction in ALS," NeuroImage: Clinical, vol. 28, Article ID 102509, 2020.

[71] C. Zhou, X. Hu, J. Hu et al., "Altered brain network in amyotrophic lateral sclerosis: a resting graph theory-based network study at voxel-wise level," Frontiers in Neuroscience, vol. 10, 2016.

[72] N. Geevasinga, M. S. Korgaonkar, P. Menon et al., "Brain functional connectome abnormalities in amyotrophic lateral sclerosis are associated with disability and cortical hyperexcitability," European Journal of Neurology, vol. 24, no. 12, pp. 1507-1517, 2017.

[73] A. Meoded, A. E. Morrissette, R. Katipally, O. Schanz, S. J. Gotts, and M. K. Floeter, "Cerebro-cerebellar connectivity is increased in primary lateral sclerosis," NeuroImage: Clinical, vol. 7, pp. 288-296, 2015.

[74] S. Chenji, S. Jha, D. Lee et al., "Investigating default mode and sensorimotor network connectivity in amyotrophic lateral sclerosis," PLoS One, vol. 11, no. 6, Article ID e0157443, 2016.

[75] J. Heimrath, M. Gorges, J. Kassubek et al., "Additional resources and the default mode network: evidence of increased connectivity and decreased white matter integrity in amyotrophic lateral sclerosis," Amyotrophic Lateral Sclerosis and Frontotemporal Degeneration, vol. 15, no. 7-8, pp. 537-545, 2014.

[76] X. Ma, J. Zhang, Y. Zhang et al., "Altered cortical hubs in functional brain networks in amyotrophic lateral sclerosis," Neurological Sciences, vol. 36, no. 11, pp. 2097-2104, 2015.

[77] E. Fortanier, A.-M. Grapperon, A. Le Troter et al., "Structural connectivity alterations in amyotrophic lateral sclerosis: a graph theory based imaging study," Frontiers in Neuroscience, vol. 13, p. 1044, 2019.

[78] M. I. Krzywinski, J. E. Schein, I. Birol et al., "Circos: an information aesthetic for comparative genomics," Genome Research, vol. 19, no. 9, pp. 1639-1645.

[79] Apparent Diffusion Coefficient and the Tensor Model-DSI Studio-a Tractography Software Tool, 2021, http://dsistudio.labsolver.org/course/apparent-diffusion-coefficientand-the-tensor-model.

[80] S. Whitfield-Gabrieli and A. Nieto-Castanon, "Conn: a functional connectivity toolbox for correlated and anticorrelated brain networks," Brain Connectivity, vol. 2, no. 3, pp. 125-141, 2012.

[81] I. S. Bakulin, A. V. Chervyakov, N. A. Suponeva, M. N. Zakharova, and M. A. Piradov, "Motor cortex hyperexcitability, neuroplasticity, and degeneration in amyotrophic lateral sclerosis," Update on Amyotrophic Lateral Sclerosis, https://www.intechopen.com/books/update-on- amyotrophic-lateral-sclerosis/motor-cortexhyperexcitability-neuroplasticity-and-degeneration-inamyotrophic-lateral-sclerosis, 2016.

[82] D. Tomasi and N. D. Volkow, "Functional connectivity density mapping," Proceedings of the National Academy of Sciences, vol. 107, no. 21, pp. 9885-9890, 2010.

[83] A. Benussi, A. Alberici, M. S. Cotelli et al., "Cortico-spinal tDCS in ALS: a randomized, double-blind, sham-controlled trial," Brain Stimulation, vol. 12, no. 5, pp. 1332-1334, 2019.

[84] M. Mazón, J. F. Vázquez Costa, A. Ten-Esteve, and L. MartíBonmatí, "Imaging biomarkers for the diagnosis and prognosis of neurodegenerative diseases. The example of amyotrophic lateral sclerosis," Frontiers in Neuroscience, vol. 12, p. 784, 2018.

[85] K. Bharti, M. Khan, C. Beaulieu et al., "Involvement of the dentate nucleus in the pathophysiology of amyotrophic lateral sclerosis: a multi-center and multi-modal neuroimaging study," NeuroImage: Clinical, vol. 28, Article ID 102385, 2020.

[86] Y. Zhang, T. Qiu, X. Yuan et al., “Abnormal topological organization of structural covariance networks in amyotrophic lateral sclerosis," NeuroImage: Clinical, vol. 21, Article ID 101619, 2019.

[87] B. Nasseroleslami, S. Dukic, M. Broderick et al., "Characteristic increases in EEG connectivity correlate with changes of structural MRI in amyotrophic lateral sclerosis," Cerebral Cortex, vol. 29, no. 1, pp. 27-41, 2019.

[88] A. P. A. Bueno, W. H. L. Pinaya, K. Rebello, L. C. de Souza, M. Hornberger, and J. R. Sato, "Regional dynamics of the resting brain in amyotrophic lateral sclerosis using fractional amplitude of low-frequency fluctuations and regional homogeneity analyses," Brain Connectivity, vol. 9, no. 4, pp. 356-364, 2019.

[89] D.-C. Shen, Y.-Y. Xu, B. Hou et al., "Monitoring value of multimodal magnetic resonance imaging in disease progression of amyotrophic lateral sclerosis," Chinese Medical Journal, vol. 131, no. 24, pp. 2904-2909, 2018.

[90] R. A. L. Menke, M. Proudfoot, K. Talbot, and M. R. Turner, "The two-year progression of structural and functional cerebral MRI in amyotrophic lateral sclerosis," NeuroImage Clin, vol. 17, pp. 953-961, 2017.

[91] F. Li, F. Zhou, M. Huang, H. Gong, and R. Xu, "Frequencyspecific abnormalities of intrinsic functional connectivity strength among patients with amyotrophic lateral sclerosis: a resting-state fMRI study," Frontiers in Aging Neuroscience, vol. 9, p. 351, 2017.

[92] H. P. Müller, M. R. Turner, J. Grosskreutz et al., "A large-scale multicentre cerebral diffusion tensor imaging study in amyotrophic lateral sclerosis," Journal of Neurology Neurosurgery and Psychiatry, vol. 87, pp. 570-579, 2016.

[93] F. Zhou, H. Gong, F. Li et al., "Altered motor network functional connectivity in amyotrophic lateral sclerosis," NeuroReport, vol. 24, no. 12, pp. 657-662, 2013. 